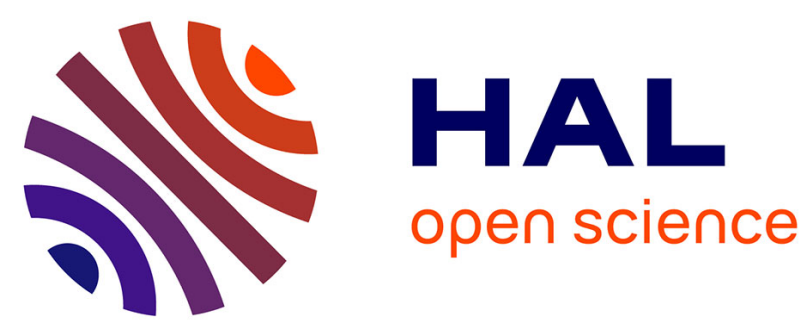

\title{
Interest rates parity and no arbitrage as equivalent equilibrium conditions in the international financial assets and goods markets
}

Stefano Bosi, Patrice Fontaine, Cuong Le Van

\section{- To cite this version:}

Stefano Bosi, Patrice Fontaine, Cuong Le Van. Interest rates parity and no arbitrage as equivalent equilibrium conditions in the international financial assets and goods markets. 2016. halshs-01391013

\section{HAL Id: halshs-01391013 \\ https://shs.hal.science/halshs-01391013}

Submitted on 2 Nov 2016

HAL is a multi-disciplinary open access archive for the deposit and dissemination of scientific research documents, whether they are published or not. The documents may come from teaching and research institutions in France or abroad, or from public or private research centers.
L'archive ouverte pluridisciplinaire HAL, est destinée au dépôt et à la diffusion de documents scientifiques de niveau recherche, publiés ou non, émanant des établissements d'enseignement et de recherche français ou étrangers, des laboratoires publics ou privés. 


\section{Documents de Travail du

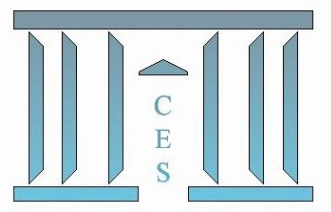

Interest rates parity and no arbitrage as equivalent equilibrium conditions in the international financial assets and goods markets

Stefano Bosi, Patrice Fontaine, Cuong LE VAN 2016.63

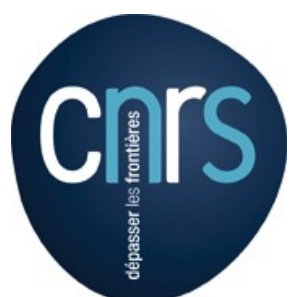


Interest rates parity and no arbitrage

as equivalent equilibrium conditions

in the international financial assets and goods markets ${ }^{*}$

Stefano Bosi

EPEE, University of Evry
Patrice Fontaine

Eurofidai, CNRS, Leonard de Vinci Research Center

Cuong Le $\operatorname{Van}^{\dagger}$

IPAG Business School, CNRS, PSE, VCREME

April 1, 2016

\begin{abstract}
In this paper, we consider a two-period consumption model with many financial assets. In the spirit of Hart [5], consumers purchase financial assets in period 0 and consume in period 1 . We differ from Hart by considering that each agent is a country. We provide conditions for the existence of an equilibrium in both international financial assets and goods markets.

First, we introduce a weaker notion of Uncovered Interest (rate) Parity (UIP) called Weak Uncovered Interest (rate) Parity (WUIP), and we show its equivalence to the no-arbitrage condition in the international financial markets.

Second, we introduce the concept of common no arbitrage and we show its equivalence to UIP.

These results bridge concepts of no arbitrage in general equilibrium theory and financial microeconomics, and of interest parity in international financial macroeconomics. In a multi-country model with many currencies and only one good, we introduce a country-specific conversion rate which transforms the returns on assets valued in local currency into units of physical good. We the define also the exchange rates between currencies of different countries. The UIP condition is required for the existence of an equilibrium in both international financial assets and goods markets and for the existence of the Law of One Price.
\end{abstract}

${ }^{*}$ We are grateful to the two Referees for their thoughtful remarks and questions. We are also grateful to Saqib Jafarey for valuable comments and to the participants of the NBER General Equilibrium Conference held in Bloomington on September 2012. This research has been conducted as part of the project LABEX MME-DII (ANR11-LBX-0023-01).

${ }^{\dagger}$ Corresponding author: levan@univ-paris1.fr. 
Keywords: general equilibrium, no arbitrage, returns on financial assets, exchange rates, Law of One Price, Uncovered Interest rate Parity.

JEL Classification: D53, F31, G11, G15. 


\section{Introduction}

In this paper, we revisit a two-period financial model by Hart [5] to introduce the international trade. The first period will be called period 0 , the second, period 1. In period 0, agents buy or sell financial assets to diversify their portfolios and maximize an expected utility function. They know the states of nature of period 1 and have beliefs on the occurrence of these states. In period 1, they buy or sell consumption goods with their endowments and the gains from financial investments. These gains are the returns on the financial assets the agents receive in each state of the nature.

In our paper, we have many assets but only one good for consumption purposes. There are many countries which have access to the same financial market. In one-country models, it is equivalent to express the returns in terms of consumption good or currency (money), while in multi-country models as ours, the prices may differ from one country to another because of the exchange rates. Therefore, we require an independent numeraire. The returns on financial assets are initially valued in local currency. However, each agent forms expectations on the conversation rates between the currency of her country and the consumption good. We introduce also the exchange rates between the currency of her country and a reference country, say country 1 . We assume that agents use their expectations on the gains of period 1 valued in terms of consumption good to purchase financial assets in period 0. Each agent maximizes a utility function in period 0 depending on her consumption in any state nature. We define the conditions for the existence of a general equilibrium of both the trade balance and the international financial market.

First, we address the issue of equilibrium existence in the international financial market. We apply the usual no-arbitrage condition of general equilibrium financial literature, which refers to the existence of appropriate financial assets prices that prevent agents from making gains without cost. In the spirit of Allouch, Le Van and Page [1], Dana, Le Van and Magnien [3], Page and Wooders [6], Werner [8] among others, we show that, under the strict concavity of utility functions, no-arbitrage conditions are necessary and sufficient to ensure the existence of an equilibrium in the international financial market.

In a second stage, we introduce two conditions. The first one is the Uncovered Interest rate Parity (UIP) which postulates the existence of exchange rates under which the returns on the financial assets of all the countries become equal in each state of nature if they are expressed in terms of consumption good. A weaker notion of UIP, called Weak Uncovered Interest rate Parity (WUIP), postulates that (i) there exists a system of exchange rates and (ii) all the countries share the same probability distribution over the states of nature such that the expected values of the returns on financial assets in terms of consumption 
good are equal. Our first main result is the equivalence of WUIP and the noarbitrage condition in the international financial market and the existence of an equilibrium in this market.

We introduce the notion of common no-arbitrage financial prices which postulates the existence of $S$ financial price vectors $q^{s}$ such that, if the gains generated by the purchases of financial assets are positive in state $s$ for any country, then the values of these assets calculated with the common no-arbitrage prices $q^{s}$ will be positive. Our second main result is to prove that UIP is equivalent to the existence of a common no-arbitrage condition.

These results bridge concepts of no arbitrage in general equilibrium theory and financial microeconomics, and of interest parity in international financial macroeconomics.

Our third result is the proof that if UIP holds then there exists an equilibrium in both financial and goods markets where the prices of financial assets are no-arbitrage prices and the prices of consumption goods in terms of numeraire satisfy the Law of One Price (LOP). Moreover, the trade balance holds in terms of nominal value and in terms of physical goods as well.

UIP implies WUIP while the converse is not true. ${ }^{1}$ Then, one may wonder what aspects of equilibrium are preserved under WUIP. While UIP entails the equilibrium in both markets, under WUIP, the equilibrium of trade balance fails. However, under the probability distribution of WUIP, the expected value of the trade balance turns out to be zero. This result is the last of the paper and sheds light on the equilibrium implications of UIP and WUIP, and their interplay.

In order to understand better the role of UIP, we provide three examples where this parity fails. In the first one, the no-arbitrage condition holds in the international financial market and an equilibrium exists but it is no-trade. In particular, the LOP is not verified. In a second example, the no-arbitrage condition holds in the international financial market and an equilibrium exists as well, but the goods market is imbalanced. In the last example, the sets of noarbitrage prices for the international financial market differ across the countries and do not intersect: thus, the no-arbitrage condition fails and there exists no equilibrium.

The paper is organized as follows. In Section 2, we introduce the main assumptions of the model, while, in Section 3 we define the equilibrium. The noarbitrage conditions in the international financial market are defined in Section 4, while some notions of parity are introduced in Section 5. In Section 6, we link the notions of no-arbitrage and UIP, and we prove two other main

\footnotetext{
${ }^{1}$ Country-specific returns change over the states of nature in the case of equities while they don't in the case of bonds. Therefore, WUIP implies UIP in the case of bonds.
} 
results of the paper, that is the equivalences (1) between the existence of a no-arbitrage financial assets price and WUIP, and (2) between the existence of common no-arbitrage condition and UIP. The existence of equilibrium is eventually proved in Section 7 through two equivalent theorems. In Theorem 1, UIP is a sufficient condition to the existence of an equilibrium. In Theorem 2, UIP is replaced by the existence of a common no-arbitrage financial assets price. In Section 8, we compare the equilibrium implications of UIP and WUIP. The implications of UIP failure are reconsidered in Section 9 through three examples. In Example 1, the only equilibrium we obtain is a no-trade equilibrium for both markets. In Example 2, UIP also fails but the no-arbitrage condition holds for the international assets market. There exists an equilibrium in the financial market but no equilibrium in the goods market. In Example 3, the no-arbitrage condition fails and no equilibrium exists in both markets. Section 10 concludes.

\section{Model}

We consider a pure exchange economy where financial assets and goods are traded in international markets. Before addressing the equilibrium issue, we introduce notations and assumptions, and we describe saving diversification and consumption.

Focus on a two-period exchange economy with many countries. Financial assets are traded in period 0 and goods are consumed in period 1 . The consumption good is supposed to be perfectly tradable. The representative agent of country $i \in\{1, \ldots, I\}$ purchases $K$ financial assets in period 0 to smooth consumption in period 1 across $S$ states of nature. At the beginning of period 1 , this agent is endowed with an amount of consumption good. This amount depends on the state of nature. Since we are in presence of many currencies, we assume the existence of a numeraire such as gold or one of the currencies. The role of this numeraire is to value the assets purchases and the consumption goods in every country.

\section{$2.1 \quad$ Notations}

For the sake of simplicity and readability, we present all the variables of the model at the beginning and currency $i$ should be understood as currency of country $i$.

We introduce the financial side of the economy through a compact notation for asset prices and quantities.

$q \equiv\left(q_{1}, \ldots, q_{K}\right)$ is a row of financial assets prices where $q_{k}$ denotes the price of asset $k$ in terms of numeraire in period 0 .

$x \equiv\left(x_{k}^{i}\right)$ is the $K \times I$ matrix of portfolios where $x_{k}^{i}$ denotes the amount of 
financial asset $k$ in the portfolio of agent $i$. Column $x^{i} \equiv\left(x_{1}^{i}, \ldots, x_{K}^{i}\right)^{T}$ is the portfolio of the representative agent $i$ of country $i$.

$R^{i} \equiv\left(R_{s k}^{i}\right)$ is the $S \times K$ matrix of returns ${ }^{2}$ where $R_{s k}^{i} \geq 0$ denotes the return on financial asset $k$ in the state of nature $s . R_{s}^{i}$ is the $s$ th row of the matrix. Returns $R_{s k}^{i}$ correspond to returns on asset $k$ in country $i$ and state $s$ and are valued in currency $i$. They are expected in period 1 with a probability $\pi_{s}^{i}$.

$\epsilon \equiv\left(\epsilon_{s}^{i}\right)$ is the $I \times S$ matrix of conversion rates where $\epsilon_{s}^{i}$ denotes the conversion rate between currency $i$ and the physical good in the state of nature $s$. Actually, one unit of physical good, in state $s$, equals $\epsilon_{s}^{i}$ units of currency $i$. Therefore, $\epsilon_{s}^{i}>0$ for any $i$ and any $s$. We denote $\epsilon^{i} \equiv\left(\epsilon_{1}^{i}, \ldots, \epsilon_{S}^{i}\right)$ the $i$ th row of the matrix. These conversion rates are actually the expected prices of commodity in terms of currency $i$. The matrix $\left(\epsilon_{s}^{i}\right)$ is exogenously given.

We obtain the expected exchange rates between currency 1 and currency $i$ by the expression $\tau_{s}^{i}=\epsilon_{s}^{1} / \epsilon_{s}^{i} . \tau \equiv\left(\tau_{s}^{i}\right)$ is the $I \times S$ matrix of exchange rates where $\tau_{s}^{i}$ denotes the exchange rate between currencies 1 and currency $i$ in the state of nature $s$. Actually, one unit of currency $i$, in state $s$, equals $\tau_{s}^{i}$ units of currency 1 . Obviously, $\tau_{s}^{i}>0$ for any $i$ and any $s$. We denote $\tau^{i} \equiv\left(\tau_{1}^{i}, \ldots, \tau_{S}^{i}\right)$ the $i$ th row of the matrix. Of course, the first row is a vector of units: $\tau_{s}^{1}=1$ for any $s .{ }^{3}$

Given the system of conversion rates $\epsilon$, let $R_{s}^{i \prime}$ denote the returns on financial assets from the country $i$ valued in physical good. More explicitly, $R_{s}^{i \prime} \equiv R_{s}^{i} / \epsilon_{s}^{i}$. These returns are also expected returns in period 1.

We introduce now a compact notation for beliefs, prices and quantities on the real side of the economy.

$c \equiv\left(c_{s}^{i}\right)$ is the $S \times I$ matrix of consumptions where $c_{s}^{i}$ denotes the amount of good consumed by agent $i$ in the state of nature $s$ in period 1. $c^{i} \equiv\left(c_{1}^{i}, \ldots, c_{S}^{i}\right)^{T}$ is the consumption column of agent $i$.

$e \equiv\left(e_{s}^{i}\right)$ is the $S \times I$ matrix of endowments where $e_{s}^{i}$ denotes the endowment nature provides to agent $i$ in the state $s$ in period $1 . e^{i} \equiv\left(e_{1}^{i}, \ldots, e_{S}^{i}\right)^{T}$ is the endowment column of agent $i$.

$\pi \equiv\left(\pi_{s}^{i}\right)$ is the $I \times S$ matrix of beliefs where $\pi_{s}^{i}$ denotes the belief of agent $i$ about the occurrence of state $s$. The individual row of beliefs $\pi^{i} \equiv\left(\pi_{1}^{i}, \ldots, \pi_{S}^{i}\right)$ lies in the $S$-unit simplex.

$p \equiv\left(p_{s}^{i}\right)$ is the $I \times S$ matrix of good prices, in period 1 . The price $p_{s}^{i}$ is the required quantity of numeraire for the purchase of one unit of good, in country $i$ and at state $s$.

\footnotetext{
${ }^{2}$ Returns are also designed as payouts or cash flows.

${ }^{3}$ We have $\tau_{s}^{i} \epsilon_{s}^{i}=\epsilon_{s}^{1}$ for any $i$. This means that, ex-ante, the commodity prices satisfy the LOP if the currency 1 is the numeraire.
} 
Notice that vectors $q, R_{s}^{i}, \tau^{i}, p^{i}, \pi^{i}$ are rows, while vectors $x^{i}, c^{i}, e^{i}$ are columns.

In the article, $\sum_{i}, \sum_{s}, \sum_{k}$ will denote unambiguously the explicit sums $\sum_{i=1}^{I}, \sum_{s=1}^{S}, \sum_{k=1}^{K}$.

\subsection{Portfolios}

The agents' behavior comes down to a saving diversification to finance future consumption. In period 1 and state $s$, agents exchange their endowments according to their portfolio:

$$
c_{s}^{i}=e_{s}^{i}+R_{s}^{i \prime} x^{i}
$$

where $R_{s}^{i \prime} \equiv R_{s}^{i} / \epsilon_{s}^{i}$. We recall that the returns $R_{s}^{i \prime}$ on portfolio are valued in terms of physical good.

Preferences of agent $i$ are rationalized by a Von Neumann-Morgenstern utility function weighted by subjective probabilities: $\sum_{s} \pi_{s}^{i} u^{i}\left(c_{s}^{i}\right)$, where $u^{i}$ is the utility function and $c_{s}^{i}$ is the quantity of her consumption good.

As a consumption amount, $c_{s}^{i}$ is required to be nonnegative and the utility function is defined on the nonnegative orthant.

Therefore, the set of portfolios is

$$
X^{i} \equiv\left\{x^{i} \in \mathbb{R}^{K}: \text { for any } s, e_{s}^{i}+R_{s}^{i \prime} x^{i} \geq 0\right\}
$$

We introduce the set of consumption allocations $Y_{+}^{i}$ generated by the purchase of a portfolio $x^{i}$ of financial assets:

$$
Y_{+}^{i} \equiv\left\{c^{i} \in \mathbb{R}^{S} \text { : there is } x^{i} \in \mathbb{R}^{K} \text { such that } c_{s}^{i}=e_{s}^{i}+R_{s}^{i \prime} x^{i} \geq 0 \text { for any } s\right\}
$$

In period 0 , given the prices of financial assets $(q)$, any agent $i$ chooses a portfolio $x^{i *}$ which maximizes her preferences taking into account the financial budget constraint:

$$
\begin{aligned}
& \max _{x^{i} \in X^{i}} \sum_{s} \pi_{s}^{i} u^{i}\left(e_{s}^{i}+R_{s}^{i \prime} x^{i}\right) \\
& q x^{i} \leq 0
\end{aligned}
$$

The RHS of the budget constraint is zero since we consider agents' net purchases.

\subsection{Assumptions}

In order to prove and characterize the existence of a general equilibrium with financial assets, we introduce some mild assumptions. The first triplet of hypotheses specifies the financial fundamentals (returns); the second triplet specifies the real fundamentals (endowments and preferences). 
Assumption 1 For any country $i$ and any state $s, \sum_{k} R_{s k}^{i}>0$.

Assumption 2 For any country $i$ and any financial asset $k, \sum_{s} R_{s k}^{i}>0$.

When Assumption 1 fails, there is a country $i$ and a state $s$ where any financial asset $k$ yields $R_{s k}^{i}=0$. In this case, the representative agent of country $i$ will consume her endowment in the state $s$.

When Assumption 2 fails, there is an asset $k$ yielding $R_{s k}^{i}=0$ in any state of nature $s$ in country $i$ : the representative agent $i$ will refuse to buy this financial asset. The following assumption is stronger and implies Assumption 2.

Assumption 3 For any country $i$ and any portfolio $x^{i} \neq 0$, the portfolio return is nonzero: $R^{i} x^{i} \neq 0$.

Assumption 3 means that there are no nonzero portfolios with a null return in any state of nature. In other terms, whatever the country $i$ we consider, the matrixes $R^{i}$ and $R^{i \prime}$ are full rank with $\operatorname{rank} R^{i}=\operatorname{rank} R^{i \prime}=K{ }^{4}$

Assumption 4 Endowments are positive: $e_{s}^{i}>0$ for any agent $i$ and any state $s$.

Assumption 5 Beliefs are positive: $\pi_{s}^{i}>0$ for any agent $i$ and any state $s$.

This assumption means that any representative agent considers each state possible.

Eventually, preferences are required to satisfy regular assumptions.

Assumption 6 For any agent $i$, the utility function $u^{i}$ is concave, continuous, strictly increasing from $\mathbb{R}_{+}$to $\mathbb{R}$.

\section{Definition of equilibrium}

Let us provide a general definition of equilibrium, then to distinguish the equilibrium in the assets markets and in the goods markets. Finally, we introduce an important and new notion of quasi-equilibrium in the sense that trade is balanced in expectations but not state of nature by state.

Definition 1 (equilibrium) Given exchange rates, beliefs and endowments $(\tau, \pi, e)$, returns and preferences $\left(R^{i}, u^{i}\right)$ for any country $i$, an equilibrium is a list of financial assets prices and allocations $(p, q, c, x)^{*}$, with $p^{*}>>0, q^{*}>>0$, and, for any $i, \sum_{s} p_{s}^{i *}=1$ and $\sum_{j} q_{j}^{*}=1$ such that: individual plans are optimal (points (1) and (2) below) and markets clear (points (3) and (4)).

\footnotetext{
${ }^{4}$ Market completeness means that the columns of $R^{i}$ span the whole space $\mathbb{R}^{S}\left(\operatorname{rank} R^{i}=S\right)$ and implies that a full insurance is possible. Redundancy of assets means that $\operatorname{dim} \operatorname{ker} R^{i}>0$, that is $K>\operatorname{rank} R^{i}$. When markets are complete and assets are not redundant, we have $K=S=\operatorname{rank} R^{i}$. In this case, the return matrix is square and invertible.
} 
(1) Portfolios are optimized given the financial assets prices $q^{*}$ : in period 0 , any agent $i$ chooses a portfolio $x^{i *}$ which maximizes her utility function under the financial budget constraint:

$$
\begin{aligned}
& \max _{x^{i} \in X^{i}} \sum_{s} \pi_{s}^{i} u^{i}\left(e_{s}^{i}+R_{s}^{i \prime} x^{i}\right) \\
& q^{*} x^{i} \leq 0
\end{aligned}
$$

(2) In period 1 , for any agent $i, c^{i *}=e^{i}+R^{i \prime} x^{i *}$ solves the program

$$
\begin{aligned}
& \max _{c^{i} \in Y_{+}^{i}} \sum_{s} \pi_{s}^{i} u^{i}\left(c_{s}^{i}\right) \\
& p^{i *} c^{i} \leq p^{i *} e^{i}
\end{aligned}
$$

(3) The international financial assets markets clear: $\sum_{i} x^{i *}=0$.

(4) The trade balance is satisfied in any state of nature: $\sum_{i} c_{s}^{i *}=\sum_{i} e_{s}^{i}$ for any $s$.

We recall that the price $p_{s}^{i}$ is the quantity of numeraire necessary for the purchase of one unit of good, in country $i$ and at state $s$. We will see later that, in equilibrium, $p^{i *} R^{i \prime} x^{i *}=q^{*} x^{i *}=0$. Therefore, in period 1 , we can actually replace the Arrow-Debreu contingent constraint $p^{i *} c^{i} \leq p^{i *} e^{i}$ by the constraint involving the income obtained with the purchase of assets in period 0 :

$$
p^{i *} c^{i} \leq p^{i *} e^{i}+p^{i *} R^{i \prime} x^{i *}
$$

An equilibrium in our economy is composed by two equilibria, one in the international assets markets and a second one in the international consumption goods market. The definitions of these equilibria are given below.

The program $\max _{x^{i} \in X^{i}} \sum_{s} \pi_{s}^{i} u^{i}\left(e_{s}^{i}+R_{s}^{i \prime} x^{i}\right)$ subject to $q x^{i} \leq 0$ is equivalent to the program $\max _{x^{i} \in X^{i}} \sum_{s} \pi_{s}^{i} u^{i}\left(e_{s}^{i}+R_{s}^{i \prime} x^{i}\right)$ subject to $p^{i}\left(e^{i}+R^{i \prime} x^{i}\right) \leq p^{i} e^{i}$ and $e^{i}+R^{i \prime} x^{i} \geq 0$. With this two-stage approach, we can link the asset prices $(q)$ to the consumption good prices $(p)$. Also, this allows us to make a distinction between the two equilibria, one for the international assets market, the other for the international goods market.

Definition 2 (equilibrium in assets markets) A list of financial assets prices and asset portfolios $(q, x)^{*}$ with $q^{*}>>0$ and $\sum_{j} q_{j}^{*}=1$, is an equilibrium for the international financial assets markets if:

(1) portfolios are optimized given the financial assets prices $q^{*}$, that is any agent $i$ chooses her portfolio $x^{i *}$ which satisfies (15);

(2) the international financial assets markets clear: $\sum_{i} x^{i *}=0$. 
Definition 3 (equilibrium in consumption goods markets) A list of consumption good prices and consumption allocations $(p, c)^{*}$ with $p^{*}>>0, \forall i, \sum_{s} p_{s}^{i *}=$ 1 , is an equilibrium for the international consumption goods markets if:

(1) consumption utilities are optimized given the consumption good prices $p^{*}$, that is any agent $i$ chooses her consumptions $c^{i *}$ which satisfies (16);

(2) the international consumption goods markets clear: $\sum_{i} c_{s}^{i *}=\sum_{i} e_{s}^{i *}$ for any $s$.

Condition (2) in the definition of equilibrium in the goods market rests on a strong requirement: the (goods) market clearing. It seems more reasonable to consider instead an international goods market which clears "at average": $\sum_{s} \delta_{s}\left[\sum_{i}\left(c_{s}^{i *}-e_{s}^{i}\right)\right]=0$, where the weight $\delta_{s}$ is just the positive probability of the state of nature $s$. In other words, the expectation of the trade balance under the probability distribution $\delta$ is null: $E_{\delta}\left[\sum_{i}\left(c^{* i}-e^{i}\right)\right]=0$. This accounts for the following notion of $\delta$-equilibrium. ${ }^{5}$

Definition 4 ( $\delta$-equilibrium) Let $\delta>>0$ be a probability distribution on the set of states of nature. Given the exchange rates, beliefs and endowments $(\tau, \pi, e)$, returns and preferences $\left(R^{i}, u^{i}\right)$ for any country $i$, a $\delta$-equilibrium is a list of financial assets prices and allocations $(p, q, c, x)^{*}$, with $p^{*}, q^{*} \neq 0$ such that: individual plans are optimal (points (1) and (2) below) and markets clear (points (3) and (4)).

(1) Portfolios are optimized given the financial assets prices $q^{*}$ : in period 0 , any agent $i$ chooses a portfolio $x^{i *}$ which maximizes her utility function under the financial budget constraint:

$$
\begin{aligned}
& \max _{x^{i} \in X^{i}} \sum_{s} \pi_{s}^{i} u^{i}\left(e_{s}^{i}+R_{s}^{i \prime} x^{i}\right) \\
& q^{*} x^{i} \leq 0
\end{aligned}
$$

(2) In period 1 , for any agent $i, c^{i *}=e^{i}+R^{i \prime} x^{i *}$ solves the program

$$
\begin{aligned}
& \max _{c^{i} \in Y_{+}^{i}} \sum_{s} \pi_{s}^{i} u^{i}\left(c_{s}^{i}\right) \\
& p^{i *} c^{i} \leq p^{i *} e^{i}
\end{aligned}
$$

(3) The international financial assets markets clear: $\sum_{i} x^{i *}=0$.

(4) The expectation under $\delta$ of the trade balance is satisfied: $E_{\delta}\left(\sum_{i} c^{* i}\right)=$ $E_{\delta}\left(\sum_{i} e^{i}\right)$.

We now provide conditions to obtain successively an equilibrium in the assets markets and an equilibrium in the international goods markets.

\footnotetext{
${ }^{5}$ In general, a $\delta$-equilibrium is not an equilibrium because trade may be imbalanced. When there is no uncertainty, $\delta$-equilibrium is equivalent to equilibrium.
} 
For assets markets, these conditions are usually called no-arbitrage conditions.

For the international goods markets, we require well-known conditions in the theory of international finance and trade: the Uncovered Interest rate Parity (UIP) and the Law of One Price (LOP). These concepts are well-defined in the case of no uncertainty. We generalize them to the stochastic case.

\section{No arbitrage in the international financial markets}

We first define the useful portfolios for every agent. The useful portfolios, called useful vectors in the general equilibrium literature, were introduced by Werner [8].

Definition 5 (useful portfolio) $w^{i}$ is a useful portfolio for agent $i$ if, for any $\mu \geq 0$ and any $x^{i} \in X^{i}$, one has:

(1) $x^{i}+\mu w^{i} \in X^{i}$,

(2) $\sum_{s} \pi_{s}^{i} u^{i}\left(e_{s}^{i}+R_{s}^{i \prime}\left(x^{i}+\mu w^{i}\right)\right) \geq \sum_{s} \pi_{s}^{i} u^{i}\left(e_{s}^{i}+R_{s}^{i \prime} x^{i}\right)$.

Let $V^{i} \equiv\left\{v^{i} \in \mathbb{R}^{K}: R_{s}^{i} v^{i} \geq 0\right.$ for any $\left.s\right\}=\left\{v^{i} \in \mathbb{R}^{K}: R_{s}^{i \prime} v^{i} \geq 0\right.$ for any $\left.s\right\}$ be the set of portfolios with nonnegative returns in any state of nature and $W^{i}$ denote the set of useful portfolios for agent $i$.

Proposition 1 Let Assumption 6 hold. For any agent $i, V^{i}=W^{i}$.

Proof: See Appendix.

We introduce a No-Arbitrage (NA) condition for financial assets markets.

Definition 6 A vector $q$ is a no-arbitrage financial assets price system (or, more compactly, a NA price) for agent $i$ if $q w^{i}>0$ for any $w^{i} \in W^{i} \backslash\{0\}$.

Condition 1 (NA) There exists a vector $q$ that is a no-arbitrage financial assets price system for any agent $i$.

Let $S^{i}$ denote the cone of no-arbitrage financial assets prices for agent $i$. The following corollary characterizes NA.

Proposition $2 N A$ is equivalent to $\cap_{i} S^{i} \neq \varnothing$.

Proof: See Appendix.

A more useful characterization of NA is given in the following proposition. 
Proposition 3 Suppose Assumptions 1, 3, 6 hold. Then $q \in \mathbb{R}^{K}$ is NA if and only if

(i) there exists a $I \times S$ matrix $\beta \equiv\left(\beta_{s}^{i}\right)$ with $\beta_{s}^{i}>0$ for any $i$ and $s$ such that $q=\sum_{s} \beta_{s}^{i} R_{s}^{i}$ for any $i$,

(ii) or, equivalently, there exists a $I \times S$ matrix $\beta^{\prime} \equiv\left(\beta_{s}^{i \prime}\right)$ with $\beta_{s}^{i \prime}>0$ for any $i$ and $s$ such that $q=\sum_{s} \beta_{s}^{i \prime} R_{s}^{i \prime}$ for any $i$.

Proof: See Appendix.

The coefficients $\beta_{s}^{i}$ can be interpreted as the prices of a unit of currency $i$, in terms of numeraire, in country $i$ and state $s$, while the coefficients $\beta_{s}^{i \prime}$ can be interpreted as the prices of a unit of good, in terms of numeraire, in country $i$ and state $s$. It is obvious that $\beta_{s}^{\prime i}=\beta_{s}^{i} \epsilon_{s}^{i}$.

We will introduce no-arbitrage financial assets prices relative to a state of nature.

Definition 7 (useful portfolio in state $s$ ) $w^{i}$ is a useful portfolio for agent $i$ in the state $s$ if, for any $\mu \geq 0$ and any $x^{i} \in X^{i}$, one has:

(1) $x^{i}+\mu w^{i} \in X^{i}$,

(2) $u^{i}\left(e_{s}^{i}+R_{s}^{i \prime}\left(x^{i}+\mu w^{i}\right)\right) \geq u^{i}\left(e_{s}^{i}+R_{s}^{i \prime} x^{i}\right)$.

Proposition $4 w^{i}$ is useful in the state $s$ if and only if $R_{s}^{i} w^{i} \geq 0$.

Proof: See Appendix.

Definition 8 A vector $q \in \mathbb{R}^{K}$ is a no-arbitrage financial assets price system for an agent $i$ in the state $s$ if $q w^{i}>0$ for any portfolio $w^{i}$ which satisfies $R_{s}^{i} w^{i}>0$ or equivalently $q w^{i}>0$ for any useful portfolio $w^{i} \neq 0$ in state $s$.

This definition relies on the no-free-lunch condition. The financial assets price $q$ is such that, if the return yielded by a portfolio $w$ is strictly positive, then its value calculated with $q$ is strictly positive.

Proposition 5 A vector $q$ is a no-arbitrage financial assets price system in the state $s$, if and only if $q=\mu_{s}^{i} R_{s}^{i}$ with $\mu_{s}^{i}>0$.

Proof: See Appendix.

Here $\mu_{s}^{i}$ is the quantity of good one obtains in country $i$ and state $s$ with one unit of currency $i$. 
Definition $9 q^{s} \in \mathbb{R}^{K}$ is a common no-arbitrage financial assets price system in the state $s$ if it is a no-arbitrage system for any $i$ in this state, i.e., for any $i$, if $R_{s}^{i} w>0$ then $q^{s} w>0$.

We say that common no-arbitrage holds for the economy if, for any state $s$, there exists a common no-arbitrage financial assets price system $q^{s}$ in this state.

Proposition 6 (1) If there exists a common no-arbitrage financial assets price system in the state $s$, then there are exchange rates $\tau_{s}^{i \prime}$ such that $\tau_{s}^{i \prime} R_{s}^{i}=R_{s}^{0}$.

(2) Conversely, if there are exchange rates $\tau_{s}^{i}$ such that $\tau_{s}^{i} R_{s}^{i}=R_{s}^{0}$ for any $i$, then there exists a common no-arbitrage financial assets price system in state $s$.

Proof: See Appendix.

Remark 1 If a common no-arbitrage financial assets price holds then there exists a NA financial assets price. Indeed, let $\left(q^{s}\right)$ be the list of $S$ common no-arbitrage financial assets price systems. Then for any $i, q^{s}=\mu_{s}^{i} R_{s}^{i}$. Let $q=\sum_{s} q^{s}$. From Proposition 3, $q$ is NA.

\section{Uncovered interest rate parities and the law of one price}

We consider two notions of parity usually applied in the theory of international finance and trade (UIP and LOP).

If we have two countries (a domestic and a foreign one), the UIP holds when the returns on domestic financial assets are equal to the returns on foreign financial assets valued in domestic currency.

Definition 10 (UIP) The Uncovered Interest rate Parity holds if there exists a system of exchange rate $\tau$ such that, for any agent $i$ and any state $s$, we have $\tau_{s}^{i} R_{s}^{i}=R_{s}^{1}$. In other words, we have $R_{s}^{i \prime}=R_{s}^{1 \prime}$.

UIP means that, the returns on the financial assets of all countries valued in consumption good, are equal for any state $s$. This condition may be considered as very stringent since it imposes that UIP holds state by state. We weaken it with the notion of Weak Uncovered Interest rate Parity (WUIP).

We introduce a convenient notation. If $\left(\delta_{s}\right)_{s=1}^{S}$ is a probability distribution over the states of nature and $\rho^{i} \equiv\left(\tau_{s}^{i} R_{s}^{i}\right)_{s=1}^{S}$ is the random vector of returns, 
then we denote its expected value by $E_{\delta}\left(\rho^{i}\right) \equiv \sum_{s=1}^{S} \delta_{s} \tau_{s}^{i} R_{s}^{i}$, that is a $K$ dimensional row.

Before stating the definition of WUIP, let us introduce a characterization.

Proposition 7 The following two statements are equivalent.

(1) There exists a probability distribution $\delta>>0$ and a system of exchange rates $\tau$ with $\tau_{s}^{1}=1$ for any $s$ such that $E_{\delta}\left(\rho^{i}\right)=E_{\delta}\left(\rho^{1}\right)$ for any $i$ with $\rho^{i} \equiv\left(\tau_{s}^{i} R_{s}^{i}\right)_{s=1}^{S}$.

(2) There exists a probability distribution $\delta^{\prime}>>0$ and a system of conversion rates $\epsilon$ such that $E_{\delta^{\prime}}\left(R^{i \prime}\right)=E_{\delta^{\prime}}\left(R^{1 \prime}\right)$ where $E_{\delta^{\prime}}\left(R^{i \prime}\right)=\sum_{s} \delta_{s}^{\prime} R_{s}^{i \prime}$ and $R_{s}^{i \prime} \equiv R_{s}^{i} / \epsilon_{s}^{i}$.

Proof: See Appendix.

This proposition allows us to introduce two equivalent definitions of Weak UIP.

Definition 11 (WUIP) (1) The Weak Uncovered Interest Rate Parity holds if there exist a common probability distribution $\left(\delta_{s}\right)_{s=1}^{S}$ with $\delta_{s}>0$ for any $s$, and a system of exchange rate $\tau$ such that $\tau_{s}^{1}=1$ for all $s$, and $E_{\delta}\left(\rho^{i}\right)=E_{\delta}\left(\rho^{1}\right)$ for any $i$ with $\rho^{i} \equiv\left(\tau_{s}^{i} R_{s}^{i}\right)_{s=1}^{S}$.

(2) Equivalently, the Weak Uncovered Interest Rate Parity holds if there exist a common probability distribution $\left(\delta_{s}\right)_{s=1}^{S}$ with $\delta_{s}>0$ for any $s$, and a system of conversion rates $\epsilon$ such that $E_{\delta^{\prime}}\left(R^{i \prime}\right)=E_{\delta^{\prime}}\left(R^{1 \prime}\right)$ for any $i$.

Proposition $8 U I P \Rightarrow W U I P$.

Proof: Apply the definitions.

Remark 2 UIP is equivalent to WUIP when there is no uncertainty.

Definition 12 (LOP) A system of consumption good prices p satisfies the Law of One Price if

$$
p_{s}^{i}=p_{s}^{1}
$$

for any $s$ and any $i$. The price of the consumption good in any country is the same when valued in currency 1 . 


\section{No-arbitrage conditions and uncovered interest rate parities: two sides of the same coin?}

The following proposition links the different notions of UIP and the noarbitrage conditions. Another main result of our paper is given just below.

Proposition 9 NA is equivalent to WUIP.

Proof: See Appendix.

Remark 3 Assume any agent $i$ has a linear utility: $u^{i}\left(c_{s}^{i}\right)=c_{s}^{i}$. The equilibrium financial assets price system is $N A$ since it satisfies $q^{*}=\lambda^{i} \pi^{i} R^{i \prime}$ with $\lambda^{i}>0$ for any $i$. It is interesting to observe that we have WUIP with specific probability distributions: the beliefs of the agents.

Proposition 10 If the return matrices $R^{i}$ and the system of exchange rates $\tau$ satisfy UIP, then NA holds.

Proof: Since UIP implies WUIP and, from Proposition 9, WUIP implies NA, the result is immediate.

Proposition 9 states the equivalence between existence of a NA financial assets price and WUIP. The following proposition claims that existence of common no-arbitrage financial assets price for the economy is equivalent to existence of exchange rates $\tau_{s}^{i}$ such that the return matrices $R^{i}$ and the system of exchange rates $\tau$ satisfy UIP. This proposition gives sufficient and necessary conditions to obtain a system of exchange rates for which UIP holds. In other words it gives sufficient and necessary conditions to endogeneize the exchange rates in order to get UIP. It is another main result of our paper.

Proposition 11 (1) If the common no-arbitrage financial assets price holds for the economy, then there are exchange rates $\tau_{s}^{i}$ such that the return matrices $R^{i}$ and the system of exchange rates $\tau$ satisfy UIP.

(2) Conversely, if there are exchange rates $\tau_{s}^{i}$ such that the return matrices $R^{i}$ and the system of exchange rates $\tau$ satisfy UIP, then common no-arbitrage financial assets price holds for the economy.

Proof: The proof follows from Proposition 6 . 


\section{Existence of equilibrium}

In this section, we prove the existence of an equilibrium in economies with international financial assets and goods markets.

\subsection{Equilibrium in the international assets markets}

We first describe a property of the equilibrium prices $q^{*}$ of financial assets markets.

Proposition 12 Let Assumptions 1, 3, 5, 6 hold. Let $(q, x)^{*}$ be an equilibrium in the financial assets markets. Then, $q^{*}$ satisfies the following equivalent properties.

(1) $q^{*}$ is NA.

(2) For any $i, q^{*}=\sum_{s} \beta_{s}^{i} R_{s}^{i}=\sum_{s} \beta_{s}^{i} \epsilon_{s}^{i} R_{s}^{i \prime}$ with $\beta_{s}^{i}>0$ for any $i$ and $s$.

Proof: See Appendix.

We now prove the existence of an equilibrium in the international financial assets market.

Proposition 13 Let Assumptions 1, 3, 4, 5, 6, hold.

(1) If NA (Condition 1) holds, then there exists an exchange rate system such that $(q, x)^{*}$ is an equilibrium in the international financial assets markets with $q_{k}^{*}>0$ for any $k$.

(2) Conversely, assume there exists an equilibrium $(q, x)^{*}$ in the international financial assets market associated with an exchange rate system. Then NA financial assets price holds.

Proof: See Appendix.

Remark 4 Observe that Proposition 13 states actually the equivalence

$N A \Leftrightarrow$ There is an equilibrium in the international financial market

Remark 5 Combining Remark 4 and Proposition 9 we have the equivalence

$W U I P \Leftrightarrow N A \Leftrightarrow$ There is an equilibrium in the international financial market 


\subsection{Equilibrium in both markets}

We come now to one of our main results.

Theorem 1 Let Assumptions 1, 3, 4, 5, 6, hold. Assume that the returns $R^{i}$ and the system of exchange rates $\tau$ satisfy UIP.

(1) There exists an equilibrium $(p, q, c, x)^{*}$ (Definition 1) with $q^{*}=\lambda p^{i *} R^{i \prime}, \lambda>$ 0 .

(2) The consumption good prices $p^{i *}$ satisfy LOP, that is $p_{s}^{i *}=p_{s}^{1 *}$ for any country $i$ and any state $s$, and there exists $\lambda>0$ such that $q^{*}=\lambda p^{i *} R^{i \prime}$ for any agent $i$.

Proof: See Appendix.

From Proposition 11, Theorem 1 is equivalent to the following theorem.

Theorem 2 Let Assumptions 1, 3, 4, 5, 6, hold. Assume the common noarbitrage financial assets price holds for the economy. Then, there exists a system of exchange rates $\tau$ for which the following statements are true.

(1) There exists an equilibrium $(p, q, c, x)^{*}$ (Definition 1) with $q^{*}=\lambda p^{i *} R^{i \prime}$ and $\lambda>0$.

(2) The consumption good prices $p^{i *}$ satisfy LOP, that is $p_{s}^{i *}=p_{s}^{1 *}$ for any country $i$ and any state $s$, and there exists $\lambda>0$ such that $q^{*}=\lambda p^{i *} R^{i \prime}$ for any agent $i$.

Remark 6 (i) It is worthwhile to notice that if the returns $R^{i}$ and the system of exchange rates $\tau$ satisfy UIP then, in equilibrium, the balance of consumption goods is satisfied also in currency 1. Explicitly, for any state $s, \sum_{i} p_{s}^{i *} c_{s}^{i *}=$ $\sum_{i} p_{s}^{i *} e_{s}^{i}$ and $\sum_{i} c_{s}^{i *}=\sum_{i} e_{s}^{i}$.

(ii) Suppose the I countries are in the same monetary zone. They use the same currency. In this case, $\tau_{s}^{i}=1$ for any $i$ and $s$. UIP implies $R_{s}^{i}=R_{s}^{1}$ and $R_{s}^{i \prime}=R_{s}^{1 \prime}$ for any $i$ and $s$.

Matrix of returns $R^{i}$ are given, but prices $(q, p)$ are endogenous. The rigidity of returns $R_{s}^{i \prime} \equiv R_{s}^{i} / \epsilon_{s}^{i}$ depends on that of the conversion rates. The equilibrium in the financial markets is ensured by the flexibility of the prices $(q, p)$, while that in the goods market depends on the conversion rates because the difference between the consumption demand and endowment is precisely given by the portfolio return. If the exchange rates satisfy UIP, then the equilibrium in the goods market is ensured. 


\section{Trade (im) balance under WUIP}

According to Theorem 1 and Remark 6, we know that, under UIP, there exists an equilibrium and the equilibrium trade is balanced, i.e., $\sum_{i} c_{s}^{i *}=\sum_{i} e_{s}^{i}$ in any state of nature $s$.

WUIP means that there exist a probability distribution $\delta$ and a system of exchange rates $\tau$ such that $\sum_{s} \delta_{s} R_{s}^{1}=\sum_{s} \delta_{s} \tau_{s}^{i} R_{s}^{i}$ for any $i$. By definition, WUIP is less demanding than UIP. We know that WUIP preserves the equilibrium in the international assets markets (Proposition 9 and 13). One may wonder what becomes the balanced trade under WUIP. While UIP implies that the value of trade balance is zero, WUIP entails instead that the expected value of trade balance under $\delta$ is zero.

We say that the returns $R^{i}$ and the system of exchange rates $\tau$ satisfy WUIP under a probability distribution $\delta$ if $\sum_{s} \delta_{s} R_{s}^{1}=\sum_{s} \delta_{s} \tau_{s}^{i} R_{s}^{i}$ for any $i$.

Theorem 3 Let Assumptions 1, 3, 4, 5, 6 hold and assume that the returns $R^{i}$ and the system of exchange rates $\tau$ satisfy WUIP under a probability distribution $\delta>>0$. Then, a $\delta$-equilibrium exists.

Proof: See Appendix.

Theorem 3 bridges the equilibrium implications of UIP and WUIP. Under WUIP, trade may be imbalanced in some state, but its expected value is zero.

A $\delta$-equilibrium is a weak notion of disequilibrium, because, precisely, trade may be imbalanced even if its expected value is zero and a financial equilibrium holds. The very reason is that, considering the expected interest parity $E_{\delta}\left(\rho^{i}\right)=E_{\delta}\left(\rho^{1}\right)$ instead of the interest parity $\rho^{i}=\rho^{1}$, we obtain the expected trade balance $E_{\delta}\left(\sum_{i} c^{i *}\right)=E_{\delta}\left(\sum_{i} e^{i}\right)$ instead of the trade balance $\sum_{i} c_{s}^{i *}=\sum_{i} e_{s}^{i}$. To understand why this happens, focus on the proof of Theorem 3: since the difference between consumption and endowment is given by the portfolio return $\left(c_{s}^{i}-e_{s}^{i}=R_{s}^{i \prime} x^{i}\right)$, if the (expected) aggregate portfolio return is zero because of the (Weak) UIP, then the (expected) aggregate net demand is zero, that is the (expected) trade balance holds.

\section{Examples with UIP failure}

In order to better understand the role of UIP, we provide three examples where UIP fails. In the first one, the no-arbitrage condition holds in the international assets market and an equilibrium exists but it is no-trade and 
the consumption goods prices don't satisfy LOP. In a second example, the noarbitrage condition holds in the international financial assets market and an equilibrium exists as well but the goods market is imbalanced. In a third example, the sets of no-arbitrage prices for the international assets market differ across the countries and do not intersect: thus, the no-arbitrage condition fails and there exists no equilibrium.

\section{Example 1}

In this example, where the UIP fails, there exists a unique equilibrium which is no-trade. We consider an exchange economy with one consumption good, two countries: $i=1,2$, two assets: $k=1,2$, and two states of nature: $s=1,2$. The matrices of returns on assets, in terms of local currencies, are given:

$$
\begin{aligned}
& R^{1}=\left(R_{s k}^{1}\right) \equiv\left[\begin{array}{ll}
R_{11}^{1} & R_{12}^{1} \\
R_{21}^{1} & R_{22}^{1}
\end{array}\right]=\left[\begin{array}{ll}
1 & 0 \\
1 & 2
\end{array}\right] \\
& R^{2}=\left(R_{s k}^{2}\right) \equiv\left[\begin{array}{ll}
R_{11}^{2} & R_{12}^{2} \\
R_{21}^{2} & R_{22}^{2}
\end{array}\right]=\left[\begin{array}{ll}
0 & 1 \\
2 & 1
\end{array}\right]
\end{aligned}
$$

In this economy, UIP is violated because $\tau_{1}^{2} R_{1}^{2}=R_{1}^{1}$ implies $\tau_{1}^{2}(0,1)=$ $(1,0)$, a contradiction.

Individuals share the same beliefs and the states are considered equiprobable:

$$
\pi \equiv\left[\begin{array}{ll}
\pi_{1}^{1} & \pi_{2}^{1} \\
\pi_{1}^{2} & \pi_{2}^{2}
\end{array}\right]=\frac{1}{2}\left[\begin{array}{ll}
1 & 1 \\
1 & 1
\end{array}\right]
$$

The utility functions are also the same across the countries:

$$
\sum_{s} \pi_{s}^{i} u^{i}\left(c_{s}^{i}\right)=\frac{1}{2} \sum_{s} \sqrt{c_{s}^{i}}
$$

but the initial endowments differ:

$$
e \equiv\left[\begin{array}{ll}
e_{1}^{1} & e_{1}^{2} \\
e_{2}^{1} & e_{2}^{2}
\end{array}\right]=\left[\begin{array}{ll}
1 & 1 \\
1 & 2
\end{array}\right]
$$

The sets of useful portfolios

$$
\begin{aligned}
& W^{1}=\left\{\left(x_{1}^{1}, x_{2}^{1}\right): x_{1}^{1} \geq 0, x_{1}^{1}+2 x_{2}^{1} \geq 0\right\} \\
& W^{2}=\left\{\left(x_{1}^{2}, x_{2}^{2}\right): x_{2}^{2} \geq 0,2 x_{1}^{2}+x_{2}^{2} \geq 0\right\}
\end{aligned}
$$

determine the cones of no-arbitrage prices:

$$
\begin{aligned}
& S^{1}=-\operatorname{int}\left(W^{1}\right)^{0}=\left\{\left(p_{1}^{1}, p_{2}^{1}\right): p_{1}^{1}>0, p_{2}^{1}>0,2 p_{1}^{1}-p_{2}^{1}>0\right\} \\
& S^{2}=-\operatorname{int}\left(W^{2}\right)^{0}=\left\{\left(p_{1}^{2}, p_{2}^{2}\right): p_{1}^{2}>0, p_{2}^{2}>0,2 p_{2}^{2}-p_{1}^{2}>0\right\}
\end{aligned}
$$


We see immediately that $(1,1) \in S^{1} \cap S^{2}$. From Proposition 13, there exists a pair $(q, x)^{*}$ with $q_{k}^{*}>0$ for any $k$ such that, for any agent $i, x^{i *}$ is a solution of program (3) and $x^{*}$ is a net trade, that is the financial assets markets clear: $\sum_{i=1}^{2} x^{i *}=0$.

In Appendix, we prove that there exists an equilibrium which is no-trade and LOP does not hold.

\section{Example 2}

In this example, UIP fails and there is no equilibrium. As above, we consider an exchange economy with one consumption good, two countries: $i=1,2$, two assets: $k=1,2$, and two states of nature: $s=1,2$. The matrix of returns $R^{1}$ remains unchanged and is given by (7), while $R^{2}$ is now replaced by a slightly different matrix:

$$
R^{2}=\left(R_{s k}^{2}\right) \equiv\left[\begin{array}{ll}
R_{11}^{2} & R_{12}^{2} \\
R_{21}^{2} & R_{22}^{2}
\end{array}\right]=\left[\begin{array}{ll}
0 & 1 \\
2 & 2
\end{array}\right]
$$

In this economy, UIP is violated because $\tau_{1}^{2} R_{1}^{2}=R_{1}^{1}$ implies $\tau_{1}^{2}(0,1)=$ $(1,0)$, a contradiction.

The other fundamentals remain the same as in Example 1 (beliefs, preferences and endowments are given by (9), (10) and (11) respectively).

Clearly, the set of useful portfolio $W^{1}$ and the cone of no-arbitrage financial assets prices $S^{1}$ do not change ( $R^{1}$ is the same), while the corresponding set for country 2 becomes now

$$
\begin{aligned}
W^{2} & =\left\{\left(x_{1}^{2}, x_{2}^{2}\right): x_{2}^{2} \geq 0,2 x_{1}^{2}+2 x_{2}^{2} \geq 0\right\} \\
S^{2} & =-\operatorname{int}\left(W^{2}\right)^{0}=\left\{\left(p_{1}^{2}, p_{2}^{2}\right): p_{1}^{2}>0, p_{2}^{2}>0, p_{2}^{2}-p_{1}^{2}>0\right\}
\end{aligned}
$$

We see immediately that $(2,3) \in S^{1} \cap S^{2}$. From Proposition 13, there exists a pair $(q, x)^{*}$ with $q_{k}^{*}>0$ for any $k$ such that, for any agent $i, x^{i *}$ is a solution of program (3) and $x^{*}$ is a net trade, that is the assets markets clear: $\sum_{i=1}^{2} x^{i *}=0$.

In Appendix we prove that the trade cannot be balanced. Hence equilibrium does not exist.

\section{Example 3}

Again, we consider an economy with two countries $i=1,2$, two financial assets $k=1,2$ and two states of nature $s=1,2$. The matrices of returns on financial assets are given:

$$
\begin{aligned}
& R^{1}=\left(R_{s k}^{1}\right) \equiv\left[\begin{array}{ll}
R_{11}^{1} & R_{12}^{1} \\
R_{21}^{1} & R_{22}^{1}
\end{array}\right]=\left[\begin{array}{ll}
1 & 0 \\
1 & 2
\end{array}\right] \\
& R^{2}=\left(R_{s k}^{2}\right) \equiv\left[\begin{array}{ll}
R_{11}^{2} & R_{12}^{21} \\
R_{21}^{2} & R_{22}^{2}
\end{array}\right]=\left[\begin{array}{ll}
1 & 4 \\
1 & 3
\end{array}\right]
\end{aligned}
$$


The sets of useful portfolios

$$
\begin{aligned}
& W^{1}=\left\{\left(x_{1}^{1}, x_{2}^{1}\right): x_{1}^{1} \geq 0, x_{1}^{1}+2 x_{2}^{1} \geq 0\right\} \\
& W^{2}=\left\{\left(x_{1}^{2}, x_{2}^{2}\right): x_{1}^{2}+4 x_{2}^{2} \geq 0, x_{1}^{2}+3 x_{2}^{2} \geq 0\right\}
\end{aligned}
$$

determine the cones of no-arbitrage financial assets prices:

$$
\begin{aligned}
& S^{1}=-\operatorname{int}\left(W^{1}\right)^{0}=\left\{\left(p_{1}^{1}, p_{2}^{1}\right): p_{2}^{1}>0,2 p_{1}^{1}-p_{2}^{1}>0\right\} \\
& S^{2}=-\operatorname{int}\left(W^{2}\right)^{0}=\left\{\left(p_{1}^{2}, p_{2}^{2}\right):-4 p_{1}^{2}+p_{2}^{2}<0,-3 p_{1}^{2}+p_{2}^{2}>0\right\}
\end{aligned}
$$

Assume there exists $\left(p_{1}, p_{2}\right) \in S^{1} \cap S^{2}$. In this case we have

$$
p_{1}>0,2 p_{1}-p_{2}>0,-4 p_{1}+p_{2}<0,-3 p_{1}+p_{2}>0
$$

These inequalities lead to $0<3 p_{1}<p_{2}<2 p_{1}$ which is impossible. Thus $S^{1} \cap S^{2}=\emptyset$ and NA financial assets price does not exist. In this case WUIP does not hold and hence UIP as well.

Remark 7 We want to show that when any country wants also to consume in period 0 then, under NA and UIP, the economy still has an equilibrium on both markets and LOP holds for any period, any state. The results concerning UIP, WUIP still hold.

Consider the model where any agent $i$ solves in period 0:

$$
\begin{aligned}
& \max _{\left(c_{0}^{i}, x^{i}\right) \in\left(\mathbb{R}_{+} \times X^{i}\right)}\left\{u_{0}^{i}\left(c_{0}^{i}\right)+\sum_{s} \pi_{s}^{i} u^{i}\left(e_{s}^{i}+R^{i \prime} x^{i}\right)\right\} \\
& p_{0}^{i}\left(c_{0}^{i}-e_{0}^{i}\right)+q x^{i} \leq 0
\end{aligned}
$$

and, in period 1 , we have $c_{s}^{i}=e_{s}^{i}+R_{s}^{i \prime} x^{i}$ for any $s$.

The functions $u_{0}^{i}$ and $u$ are strictly concave and increasing.

An equilibrium of this economy is a list $(p, q, c, x)^{*}$ with $p^{*}, q^{*}>>0$ such that individual plans are optimal (points (1) and (2) below) and markets clear (points (3) and (4)).

(1) Portfolios and consumption in period 0 are optimized given the financial assets prices and consumption price $q^{*}$ and $p_{0}^{*}$ : in period 0 , any agent $i$ chooses a consumption $c_{0}^{i *}$ and a portfolio $x^{i *}$ which maximizes her utility function under the budget constraint:

$$
\begin{aligned}
& \max _{\left(c_{0}^{i}, x^{i}\right) \in\left(\mathbb{R}_{+} \times X^{i}\right)}\left\{u_{0}^{i}\left(c_{0}^{i}\right)+\sum_{s} \pi_{s}^{i} u^{i}\left(e_{s}^{i}+R_{s}^{i \prime} x^{i}\right)\right\} \\
& p_{0}^{i *}\left(c_{0}^{i}-e_{0}^{i}\right)+q^{*} x^{i} \leq 0
\end{aligned}
$$


(2) In period 1 , for any agent $i, c^{i *}=e^{i}+R^{i \prime} x^{i *}$ solves the program

$$
\begin{aligned}
& \max _{c^{i} \in Y_{+}^{i}} \sum_{s} \pi_{s}^{i} u^{i}\left(c_{s}^{i}\right) \\
& p^{i *} c^{i} \leq p^{i *} e^{i}
\end{aligned}
$$

(3) The international financial assets markets clear: $\sum_{i} x^{i *}=0$.

(4) The trade balance is satisfied in period 0 and in any state of nature of period 1:

$$
\begin{aligned}
\sum_{i} c_{0}^{i *} & =\sum_{i} e_{0}^{i} \\
\sum_{i} c_{s}^{i *} & =\sum_{i} e_{s}^{i} \text { for } s=1, \ldots, S
\end{aligned}
$$

Claim 1 Assume UIP hold.

(1) There exists an equilibrium $(p, q, c, x)^{*}$ with $q^{*}=p^{i *} R^{i \prime}$, for any $i$.

(2) The consumption good prices $p^{i *}$ satisfy LOP, that is

(a) in period $0, p_{0}^{i *}=p_{0}^{1 *}$, for any country $i$,

(b) in period $1, p_{s}^{i *}=p_{s}^{1 *}$ for any country $i$ and any state $s$,

Proof. Define $p_{0}^{i}=p_{0}$ for any $i$, where $p_{0} \geq 0$. At period 0 agent $i$ solves

$$
\begin{aligned}
& \max _{\left(c_{0}^{i}, x^{i}\right) \in\left(\mathbb{R}_{+} \times X^{i}\right)}\left\{u_{0}^{i}\left(c_{0}^{i}\right)+\sum_{s} \pi_{s}^{i} u^{i}\left(e_{s}^{i}+R_{s}^{i \prime} x^{i}\right)\right\} \\
& p_{0}\left(c_{0}^{i}-e_{0}^{i}\right)+q x^{i} \leq 0
\end{aligned}
$$

Recall that $S^{i}$ denotes the set of no-arbitrage asset prices for agent $i$. Then, the set of no-arbitrage prices for period 0 is $\sigma=\mathbb{R}_{++} \times \cap_{i} S^{i}$. In addition, $\cap_{i} S^{i} \neq \varnothing \Leftrightarrow \sigma \neq \varnothing$. From e.g. Dana, Le Van and Magnien, 1999, there exists $\left(p_{0}, q,\left(c_{0}^{i}, x^{i}\right)_{i}\right)^{*}$ with $\left(p_{0}, q\right)^{*}>>0$, such that any agent $i$ solves her $/$ his consumption problem with prices $\left(p_{0}, q\right)^{*}$ and $\sum_{i}\left(c_{0}^{i *}-e_{0}^{i *}\right)=0, \sum_{i} x_{0}^{i *}=0$. For period 1, the proof of Theorem 1 applies.

We can easily check that we still have $N A \Leftrightarrow W U I P$ since these results are relative only to the second period.

\section{Conclusion}

Our paper considers a two-period model à la Hart [5] with consumption good and financial assets. The consumption and the assets are internationally tradable. Our main results are the following. 
(1) We introduce a Weak UIP condition and we show that it is equivalent to No-Arbitrage Condition financial assets price. Moreover we have the equivalences:

\author{
NA holds $\Leftrightarrow \quad$ Weak UIP holds \\ $\Leftrightarrow \quad$ An equilibrium exists in the international financial markets
}

(2) We introduce the common no-arbitrage financial assets price and show

Existence of a common no-arbitrage financial asset price $\Leftrightarrow$ UIP holds

(3) When the UIP holds, we have an equilibrium in both the international financial assets and goods markets. At this equilibrium, we obtain trade balance in value and LOP.

(4) When the UIP fails, we show by means of three examples that (i) the no-arbitrage financial assets price condition holds and an equilibrium exists as well but it is no-trade and LOP is not satisfied (Example 1); (ii) the noarbitrage condition price holds in the international financial assets market and an equilibrium exists as well but there exists no balance in the goods markets (Example 2); (iii) the no-arbitrage condition financial assets price fails and there exists no equilibrium (Example 3 ).

Modelling the coexistence of an equilibrium in the financial assets markets jointly with a disequilibrium in the goods markets through the failure of parities remains an interesting question. Most of financial papers (Rogoff [7] among others) consider that the parities are not respected in the short run. As suggested by Frenkel and Mussa [4] in a monetary model, trade balance disequilibria seem plausible under a regime of pegged rates because relative price adjustments are achieved through slow changes in the goods markets, while financial markets are mobile and integrated. This point is tackled in the paper through three examples.

\title{
11 Appendix
}

\section{Proof of Proposition 1}

First, we want to prove that $V^{i} \subseteq W^{i}$. If $v^{i} \in V^{i}, R_{s}^{i \prime} v^{i} \geq 0$ for any $s$. According to (2), for any $x^{i} \in X^{i}$ and any $\mu \geq 0$, one has $e_{s}^{i}+R_{s}^{i \prime}\left(x^{i}+\mu v^{i}\right)=$ $e_{s}^{i}+R_{s}^{i \prime} x^{i}+\mu R_{s}^{i \prime} v^{i} \geq \mu R_{s}^{i \prime} v^{i} \geq 0$, that is Definition 5, point (1). From

$$
e_{s}^{i}+R_{s}^{i \prime}\left(x^{i}+\mu v^{i}\right)=e_{s}^{i}+R_{s}^{i \prime} x^{i}+\mu R_{s}^{i \prime} v^{i} \geq e_{s}^{i}+R_{s}^{i \prime} x^{i}
$$

and the increasingness of $u^{i}$ (Assumption 6), we obtain also Definition 5, point (2). 
Conversely, we want to show that $W^{i} \subseteq V^{i}$. Let $w^{i} \in W^{i}$. Then, point (1) in Definition 5 requires $e_{s}^{i}+R_{s}^{i \prime}\left(x^{i}+\mu w^{i}\right) \geq 0$ for any $s$. Dividing both the sides of this inequality by $\mu>0$ and letting $\mu$ go to infinity, we get $R_{s}^{i \prime} w^{i} \geq 0$ for any $s$, that is $w^{i} \in V^{i}$.

Finally, observe $R_{s}^{i \prime} w^{i} \geq 0 \Leftrightarrow R_{s}^{i} w^{i} \geq 0$.

\section{Proof of Proposition 2}

We observe that $S^{i}=-\operatorname{int}\left(W^{i}\right)^{0}$ where $\left(W^{i}\right)^{0}$ is the polar ${ }^{6}$ of $W^{i}$. Under Assumption 3, the sets $W^{i}$ do not contain lines and the sets $S^{i}$ are nonempty (see Dana, Le Van and Magnien [3] among others). Let $\cap_{i} S^{i}$ be the intersection of all the cones of no-arbitrage prices. Thus, if NA holds, $q$ belongs to $\cap_{i} S^{i}$, and, if $\cap_{i} S^{i}$ is nonempty, NA holds.

\section{Proof of Proposition 3}

(1) Let $q$ be NA. Let $w^{i} \in W^{i} \backslash\{0\}$. From Assumption $3, R_{s}^{i} w^{i} \geq 0$ for any $s$ and $R_{s}^{i} w^{i}>0$ for some $s$. From Dana and Jeanblanc [2], there exists a $I \times S$ matrix $\beta \equiv\left(\beta_{s}^{i}\right)$ with $\beta_{s}^{i}>0$ for any $i$ and $s$ such that $q=\sum_{s} \beta_{s}^{i} R_{s}^{i}$ for any $i$.

(2) Conversely, assume $q=\sum_{s} \beta_{s}^{i} R_{s}^{i}$ for any $i$ with $\beta_{s}^{i}>0$ for any $i$ and $s$. If $w^{i} \in W^{i} \backslash\{0\}$, then, from Assumption $3, R_{s}^{i} w \geq 0$ for any $s$ and $R_{s}^{i} w>0$ for some $s$. Hence, for any $i, q w^{i}>0$ for any $w^{i} \in W^{i} \backslash\{0\}$ or, equivalently, $q$ is NA.

\section{Proof of Proposition 4}

Sufficiency. $R_{s}^{i} w^{i} \geq 0$ implies $u^{i}\left(e_{s}^{i}+R_{s}^{i \prime}\left(x^{i}+\mu w^{i}\right)\right)=u^{i}\left(e_{s}^{i}+R_{s}^{i \prime} x^{i}+\mu R_{s}^{i \prime} w^{i}\right) \geq$ $u^{i}\left(e_{s}^{i}+R_{s}^{i \prime} x^{i}\right)$ because $u^{i}$ is increasing.

Necessity. $u^{i}$ is concave and $w^{i}$ is useful in the state $s$. Then,

$$
u^{i \prime}\left(e_{s}^{i}+R_{s}^{i \prime} x^{i}\right) \mu R_{s}^{i \prime} w^{i} \geq u^{i}\left(e_{s}^{i}+R_{s}^{i \prime} x^{i}+\mu R_{s}^{i \prime} w^{i}\right)-u^{i}\left(e_{s}^{i}+R_{s}^{i \prime} x^{i}\right) \geq 0
$$

Dividing both the sides of this inequality by $\mu u^{i \prime}\left(e_{s}^{i}+R_{s}^{i \prime} x^{i}\right)>0$, we obtain $R_{s}^{i \prime} w^{i} \geq 0$. It is equivalent to $R_{s}^{i} w^{i} \geq 0$.

\section{Proof of Proposition 5}

$R_{s}^{i} w^{i} \geq 0$ implies that $w^{i}$ is useful in the state $s$ and, since $q$ is a no-arbitrage asset price system in the state $s, q w^{i} \geq 0$. According to the Farkas' Lemma, $\left(R_{s}^{i} w^{i}>0 \Rightarrow q w^{i}>0\right)$ implies $q=\mu_{s}^{i} R_{s}^{i}$ with $\mu_{s}^{i}>0$.

The converse is obvious.

\section{Proof of Proposition 6}

(1) Since $q^{s}=\mu_{s}^{i} R_{s}^{i}=\mu_{s}^{1} R_{s}^{1}$ for any $i$, we can define $\tau_{s}^{i} \equiv \mu_{s}^{i} / \mu_{s}^{1}$ in order to obtain $\tau_{s}^{i} R_{s}^{i}=R_{s}^{1}$.

\footnotetext{
${ }^{6}$ The polar cone of a set $X \subseteq \mathbb{R}^{K}$ is defined as $X^{0} \equiv\left\{y \in \mathbb{R}^{K}: y^{T} x \leq 0\right.$ for any $\left.x \in X\right\}$.
} 
(2) Consider a useful portfolio $w^{i} \neq 0$ which satisfies $R_{s}^{i} w^{i}>0$. Define $q^{s} \equiv R_{s}^{1}=\tau_{s}^{i} R_{s}^{i}$. Then, $q^{s} w^{i}>0$ for any $i$. Apply Definitions 8 and 9 .

\section{Proof of Proposition 7}

$(1) \Rightarrow(2)$. Assume we have (1). Take $\left(\epsilon_{s}^{1}\right)_{s}>>$ 0. Define $R_{s}^{i \prime} \equiv R_{s}^{i} / \epsilon_{s}^{i}$, $\epsilon_{s}^{i}=\epsilon_{s}^{1} / \tau_{s}^{i}$ and

$$
\delta_{s}^{\prime} \equiv \frac{\delta_{s} \epsilon_{s}^{1}}{\sum_{\sigma} \delta_{\sigma} \epsilon_{\sigma}^{1}}
$$

We get $\sum_{s} \delta_{s}^{\prime} R_{s}^{i \prime}=\sum_{s} \delta_{s}^{\prime} R_{s}^{1 \prime}$, that is (2).

$(2) \Rightarrow(1)$. Define $\tau_{s}^{i}=\epsilon_{s}^{1} / \epsilon_{s}^{i}$ and

$$
\delta_{s} \equiv \frac{\delta_{s}^{\prime} / \epsilon_{s}^{1}}{\sum_{\sigma} \delta_{\sigma}^{\prime} / \epsilon_{\sigma}^{1}}
$$

Then $E_{\delta^{\prime}}\left(R^{i \prime}\right)=E_{\delta^{\prime}}\left(R^{1 \prime}\right)$ becomes $\sum_{s} \delta_{s} \tau_{s}^{i} R_{s}^{i}=\sum_{s} \delta_{s} R_{s}^{1}$, that is (1).

\section{Proof of Proposition 9}

(1) If a vector $q$ is NA, then from Proposition 3 , there exists $\beta_{s}^{i}>0$ for any $i$ and $s$ such that $q=\sum_{s} \beta_{s}^{i} R_{s}^{i}$ for any $i$. Define $\delta_{s} \equiv \beta_{s}^{1} / \sum_{\sigma} \beta_{\sigma}^{1}$ and $\tau_{s}^{i} \equiv \beta_{s}^{i} / \beta_{s}^{1}$. Clearly, the probability distribution $\delta$ is well-defined. Consider the random vectors $\rho^{1} \equiv\left(R_{s}^{1}\right)_{s=1}^{S}$ and $\rho^{i} \equiv\left(\tau_{s}^{i} R_{s}^{i}\right)_{s=1}^{S}$. Then,

$$
E_{\delta}\left(\rho^{1}\right)=\sum_{s=1}^{S} \delta_{s} R_{s}^{1}=\frac{\sum_{s=1}^{S} \beta_{s}^{1} R_{s}^{1}}{\sum_{s} \beta_{s}^{1}}=\frac{\sum_{s=1}^{S} \beta_{s}^{i} R_{s}^{i}}{\sum_{s} \beta_{s}^{1}}=\sum_{s=1}^{S} \frac{\beta_{s}^{1}}{\sum_{\sigma} \beta_{\sigma}^{1}} \tau_{s}^{i} R_{s}^{i}=E_{\delta}\left(\rho^{i}\right)
$$

that is WUIP.

(2) Conversely, if $E_{\delta}\left(\rho^{i}\right)=E_{\delta}\left(\rho^{1}\right)$ for any $i$, then the vector

$$
q=E_{\delta}\left(\rho^{1}\right)=E_{\delta}\left(\rho^{i}\right)=\sum_{s=1}^{S} \delta_{s} \tau_{s}^{i} R_{s}^{i}, \text { for any } i
$$

is NA from Proposition 3.

\section{Proof of Proposition 12}

(1) First observe that we have $q^{*} x^{i *}=0$ for all $i$. Let $\bar{\varepsilon}=(\varepsilon, \ldots, \varepsilon)^{T} \in \mathbb{R}^{K}$ with $\varepsilon>0$. Let $w \in W^{i}$. We then have

$$
\begin{aligned}
\sum_{s} \pi_{s}^{i} u^{i}\left(e_{s}^{i}+R_{s}^{i \prime}\left(x^{i *}+w^{i}+\bar{\varepsilon}\right)\right) & >\sum_{s} \pi_{s}^{i} u^{i}\left(e_{s}^{i}+R_{s}^{i \prime}\left(x^{i *}+w^{i}\right)\right) \\
& \geq \sum_{s} \pi_{s}^{i} u^{i}\left(e_{s}^{i}+R_{s}^{i \prime} x^{i *}\right)
\end{aligned}
$$

which implies $q^{*}\left(w^{i}+\bar{\varepsilon}\right)>0$. Let $\varepsilon$ go to zero. We get $q^{*} w^{i} \geq 0$. Now, let $w^{i} \in W^{i} \backslash\{0\}$. Then $R_{s}^{i} w^{i} \geq 0$ for all $s$. However, from Assumption 3, there exists $s$ with $R_{s}^{i} w^{i}>0$. Then, from Assumption 5, we have, for any $i$,

$$
\sum_{s} \pi_{s}^{i} u^{i}\left(e_{s}^{i}+R_{s}^{i \prime}\left(x^{i *}+w^{i}\right)\right)>\sum_{s} \pi_{s}^{i} u^{i}\left(e_{s}^{i}+R_{s}^{i \prime} x^{i *}\right)
$$


This implies $q^{*} w^{i}>0$ and $q^{*} \in S^{i}$ for any $i$. Equivalently, $q^{*}$ is NA.

(2) The second statement comes from Proposition 3.

\section{Proof of Proposition 13}

(1) Assume that $q^{*}$ is NA. In this case, the proof is provided by Werner [8], Page and Wooders [6], and Dana, Le Van, Magnien [3] among others. The strict positivity of $q^{*}$ results from the strict increasingness of $u^{i}$ jointly with Assumptions 1 and 3.

(2) Refer to Proposition 12.

\section{Proof of Theorem 1}

(1) Since we have UIP, the price $q=\sum_{s} R_{s}^{i \prime}$ is NA since it equals $\sum_{s} R_{s}^{1 \prime}$. It follows from Proposition 13 that we have an equilibrium $\left(\tilde{q}, x^{*}\right)$ in the international assets markets.

From Proposition $12, \tilde{q}^{*}=\sum_{s} \beta_{s}^{i \prime} R_{s}^{i \prime}$ for any $i$, with $\beta_{s}^{i \prime}>0$ for any $i$ and any $s$. Define for any $i$

$$
\tilde{\beta}_{s}^{i} \equiv \frac{\beta_{s}^{i \prime}}{\sum_{\sigma} \sum_{k} \beta_{\sigma}^{i \prime} R_{\sigma k}^{i \prime}}
$$

We claim that, for any $i, \sum_{s} \tilde{\beta}_{s}^{i \prime} R_{s}^{i \prime}=\sum_{s} \tilde{\beta}_{s}^{1 \prime} R_{s}^{1 \prime}$. Indeed, for any $k$ and any $i$, we have $\sum_{s} \beta_{s}^{i \prime} R_{s k}^{i \prime}=\sum_{s} \beta_{s}^{1 \prime} R_{s k}^{1 \prime}$. Hence, for any $i, \sum_{\sigma} \sum_{k} \beta_{\sigma}^{i \prime} R_{\sigma k}^{i \prime}=$ $\sum_{\sigma} \sum_{k} \beta_{\sigma}^{1 \prime} R_{\sigma k}^{1 \prime}$. Let $\lambda \equiv \sum_{\sigma} \sum_{k} \beta_{\sigma}^{1 \prime} R_{\sigma k}^{1 \prime}=\sum_{\sigma} \sum_{k} \beta_{\sigma}^{i \prime} R_{\sigma k}^{i \prime}$ for any $i$. Hence,

$$
\sum_{s} \frac{\beta_{s}^{i \prime} R_{s}^{i \prime}}{\lambda}=\sum_{s} \frac{\beta_{s}^{1 \prime} R_{s}^{1 \prime}}{\lambda}
$$

Equivalently,

$$
\sum_{s} \frac{\beta_{s}^{i \prime} R_{s}^{i \prime}}{\sum_{\sigma} \sum_{k} \beta_{\sigma}^{i \prime} R_{\sigma k}^{i \prime}}=\sum_{s} \frac{\beta_{s}^{1 \prime} R_{s}^{1 \prime}}{\sum_{\sigma} \sum_{k} \beta_{\sigma}^{1 \prime} R_{\sigma k}^{1 \prime}}
$$

Our claim is true.

Now, define $q^{*} \equiv \sum_{s} \tilde{\beta}_{s}^{i} R_{s}^{i \prime}$. We have $\sum_{j} q_{j}^{*}=1$.

Setting $\tilde{p}_{s}^{i} \equiv \tilde{\beta}_{s}^{i}$, for all $i$, all $s$, we find

$$
q^{*}=\sum_{s} \tilde{p}_{s}^{i} R_{s}^{i \prime}=\sum_{s} \tilde{p}_{s}^{i} R_{s}^{1 \prime}=\sum_{s} \tilde{p}_{s}^{1} R_{s}^{1 \prime}
$$

for any $i$. Let $Z \equiv\left\{z \in \mathbb{R}^{S}: \sum_{s} z_{s} R_{s}^{1 \prime}=0\right\}$ and observe that $Z=\{0\}$ in the case of complete assets markets $\left(\operatorname{rank} R^{1 \prime}=S\right)$.

From (18), we get, for any $i, \tilde{p}_{s}^{i}=\tilde{p}_{s}^{1}+z_{s}^{i}$ with $z^{i} \in Z$. Define $\hat{p}_{s}^{1} \equiv \tilde{p}_{s}^{1}$ and $\hat{p}_{s}^{i} \equiv \tilde{p}_{s}^{i}-z_{s}^{i}=\hat{p}_{s}^{1}$ for any $i$ and any $s$. Let $c_{s}^{i *}=e_{s}^{i}+R_{s}^{i \prime} x^{i *}$ for any $i$ and any s. $q^{*} x^{i *}=0$ implies that $\hat{p}^{i} c^{i *}=\hat{p}^{i} e^{i}+q^{*} x^{i *}=\hat{p}^{i} e^{i}$ : the budget constraint is satisfied in any country (Definition 1, point (2)).

Noticing that, for any $i$ and $s, c_{s}^{i *}=e_{s}^{i *}+R_{s}^{i \prime} x^{i *}=e_{s}^{i *}+R_{s}^{1 \prime} x^{i *}$ and summing over $i$, we obtain for any $s$

$$
\sum_{i} c_{s}^{i *}=\sum_{i} e_{s}^{i *}+R_{s}^{1 \prime} \sum_{i} x^{i *}=\sum_{i} e_{s}^{i *}
$$


since $\sum_{i} x^{i *}=0$.

We notice that

$$
q^{*}=\sum_{s} \tilde{p}_{s}^{i} R_{s}^{i \prime}=\sum_{s} \hat{p}_{s}^{1} R_{s}^{i \prime}+\sum_{s} z_{s}^{i} R_{s}^{i \prime}=\sum_{s} \hat{p}_{s}^{i} R_{s}^{i \prime}+\sum_{s} z_{s}^{i} R_{s}^{1 \prime}=\sum_{s} \hat{p}_{s}^{i} R_{s}^{i \prime}
$$

because $z^{i} \in Z$.

We will show that $\left(\hat{p}, c^{*}\right)$ satisfies the point $(2)$ of Definition 1. For that, define $c_{s}^{i}=e_{s}^{i}+R_{s}^{i \prime} x^{i}$ and assume that

$$
\sum_{s} \pi_{s}^{i} u^{i}\left(e_{s}^{i}+R_{s}^{i \prime} x^{i}\right)>\sum_{s} \pi_{s}^{i} u^{i}\left(e_{s}^{i}+R_{s}^{i \prime} x^{i *}\right)
$$

Since $(q, x)^{*}$ is an equilibrium in the international assets markets, we have $q^{*} x^{i}>q^{*} x^{i *}$. Equivalently, $\sum_{s} \hat{p}_{s}^{i} R_{s}^{i \prime} x^{i}>\sum_{s} \hat{p}_{s}^{i} R_{s}^{i \prime} x^{i *}$ and hence

$$
\sum_{s} \hat{p}_{s}^{i *} c_{s}^{i}=\sum_{s} \hat{p}_{s}^{i} e_{s}^{i}+\sum_{s} \hat{p}_{s}^{i} R_{s}^{i \prime} x^{i}>\sum_{s} \hat{p}_{s}^{i} e_{s}^{i}+\sum_{s} \hat{p}_{s}^{i} R_{s}^{i \prime} x^{i *}=\sum_{s} \hat{p}_{s}^{i} e_{s}^{i}
$$

We have proved that $\left(\hat{p}, c^{*}\right)$ satisfies point $(2)$ of Definition 1.

Observe that $\hat{p}>>0$. We now normalize the equilibrium consumption prices by defining

$$
p_{s}^{i *}=\frac{\hat{p}_{s}^{i}}{\sum_{\sigma} \hat{p}_{\sigma}^{i}}
$$

for any $i$ and any $s$.

Let $\lambda \equiv 1 / \sum_{\sigma} \hat{p}_{\sigma}^{i}$. Then, $q^{*}=\lambda p^{i *} R^{i \prime}$ for any $i$.

(2) Finally, it is clear that the system $p^{*}$ satisfies the LOP.

\section{Proof of Theorem 3}

Since the returns $R^{i}$ and the system of exchange rates $\tau$ satisfy WUIP under a probability distribution $\delta>>0$, no-arbitrage condition (NA) holds. Hence an equilibrium in the international assets markets $(q, x)^{*}$ exists. If $c^{*}$ denotes the associated consumptions in period 1 , we have $c_{s}^{i *}=e_{s}^{i}+\tau_{s}^{i} R_{s}^{i} x^{i *}$ for any $i$ and $s$. We can easily check that they satisfy the consumers problems (16). We now check that the expectation under $\delta$ of the trade balance is satisfied. Indeed, we have

$$
\begin{aligned}
\sum_{s} \delta_{s} \sum_{i} c_{s}^{i *} & =\sum_{s} \delta_{s} \sum_{i} e_{s}^{i}+\sum_{i}\left(\sum_{s} \delta_{s} \tau_{s}^{i} R_{s}^{i}\right) x^{i *} \\
& =\sum_{s} \delta_{s} \sum_{i} e_{s}^{i}+\sum_{i}\left(\sum_{s} \delta_{s} R_{s}^{1}\right) x^{i *} \\
& =\sum_{s} \delta_{s} \sum_{i} e_{s}^{i}+\left(\sum_{s} \delta_{s} R_{s}^{1}\right) \sum_{i} x^{i *} \\
& =\sum_{s} \delta_{s} \sum_{i} e_{s}^{i}
\end{aligned}
$$


since $\sum_{i} x^{i *}=0$.

\section{Example 1}

Trade balances are satisfied in any state of nature $s$ if $\sum_{i} c_{s}^{i *}=\sum_{i} e_{s}^{i}$.

Let

$$
\tau \equiv\left(\tau_{s}^{i}\right)=\left[\begin{array}{cc}
1 & 1 \\
\tau_{1}^{1} & \tau_{2}^{1}
\end{array}\right]
$$

be the $I \times S$ matrix of exchange rates, $R_{s}^{1 \prime}=R_{s}^{1} / \epsilon_{s}^{1}$ and $R_{s}^{2 \prime}=R_{s}^{2} / \epsilon_{s}^{2}$ and $\tau_{s}^{2}=$ $\epsilon_{s}^{1} / \epsilon_{s}^{2}$. From equation (1), we know that $c_{s}^{i *}-e_{s}^{i}=R_{s}^{i \prime} x^{i *}$. Thus, $\sum_{i} R_{s}^{i \prime} x^{i *}=0$ for any $s$. In our example, we get

$$
\begin{aligned}
\frac{R_{11}^{1}}{\epsilon_{1}^{1}} x_{1}^{1 *}+\frac{R_{12}^{1}}{\epsilon_{1}^{1}} x_{2}^{1 *}+\frac{R_{11}^{2}}{\epsilon_{1}^{2}} x_{1}^{2 *}+\frac{R_{12}^{2}}{\epsilon_{1}^{2}} x_{2}^{2 *} & =0 \\
\text { or } R_{11}^{1} x_{1}^{1 *}+R_{12}^{1} x_{2}^{1 *}+\tau_{1}^{2} R_{11}^{2} x_{1}^{2 *}+\tau_{1}^{2} R_{12}^{2} x_{2}^{2 *} & =0
\end{aligned}
$$

Using (7) and (8) to replace $R_{11}^{1}, R_{11}^{1}, R_{11}^{2}$ and $R_{12}^{2}$ by their numerical values, we find $x_{1}^{1 *}+\tau_{1}^{2} x_{2}^{2 *}=0$. Since $x_{2}^{1 *}+x_{2}^{2 *}=0$, we obtain also $x_{1}^{1 *}-\tau_{1}^{2} x_{2}^{1 *}=0$, that is a contradiction with $q_{1}^{*} x_{1}^{1 *}+q_{2}^{*} x_{2}^{1 *}=0$ under price positivity if $x_{1}^{1 *} \neq 0$. Hence $x_{1}^{1 *}=0$. In this case, we have $x_{1}^{2 *}=x_{2}^{2 *}=0$. If this allocation is an equilibrium, it solves (3): there are positive multipliers $\mu^{i *}$ such that $q_{k}^{*}=$ $\mu^{i *} \sum_{s} \pi_{s}^{i} u^{\prime}\left(c_{s}^{i *}\right) R_{s k}^{i \prime}$ for any $k$ and $i=1,2$. Noticing that $c_{s}^{i *}=e_{s}^{i}+R_{s 1}^{i \prime} x_{1}^{i *}+$ $R_{s 2}^{i \prime} x_{2}^{i *}$ and $\left(c_{1}^{1}, c_{2}^{1}, c_{1}^{2}, c_{2}^{2}\right)^{*}=(1,1,1,2)$, we find

$$
\begin{aligned}
& q_{1}^{*}=\frac{\mu^{1 *}}{2} \sum_{s} \frac{\pi_{s}^{1} R_{s 1}^{1}}{\sqrt{c_{s}^{1 *}}}=\frac{\mu^{1 *}}{2} \text { and } q_{1}^{*}=\frac{\mu^{2 *}}{2} \sum_{s} \frac{\pi_{s}^{2} R_{s 1}^{2 \prime}}{\sqrt{c_{s}^{2 *}}}=\frac{\mu^{2 *}}{2} \frac{\tau_{2}^{2}}{\sqrt{2}} \\
& q_{2}^{*}=\frac{\mu^{1 *}}{2} \sum_{s} \frac{\pi_{s}^{1} R_{s 2}^{1}}{\sqrt{c_{s}^{1 *}}}=\frac{\mu^{1 *}}{2} \text { and } q_{2}^{*}=\frac{\mu^{2 *}}{2} \sum_{s} \frac{\pi_{s}^{2} R_{s 2}^{2 \prime}}{\sqrt{c_{s}^{2 *}}}=\frac{\mu^{2 *}}{4}\left(\tau_{1}^{2}+\frac{\tau_{2}^{2}}{\sqrt{2}}\right)
\end{aligned}
$$

This implies $\tau_{2}^{2}=\tau_{1}^{2} \sqrt{2}$. Thus, we then get a no-trade equilibrium with, for any $i, x^{i *}=0$ and $c^{i *}=e^{i}$. To compute the prices, we assume, for simplicity, that $\epsilon_{1}^{1}=\epsilon_{2}^{1}=1$. Prices are given by $q^{*}=(1,1)$ and, using $q^{*}=p^{i *} R^{i \prime}$,

$$
p^{*} \equiv\left[\begin{array}{cc}
p_{1}^{1} & p_{2}^{1} \\
p_{1}^{2} & p_{2}^{2}
\end{array}\right]^{*}=\frac{1}{2}\left[\begin{array}{cc}
1 & 1 \\
\frac{1}{\tau_{1}^{2}} & \frac{1}{\tau_{2}^{2}}
\end{array}\right]
$$

We observe that $p_{1}^{1} \neq p_{1}^{2}$ and $p_{2}^{1} \neq p_{2}^{2}$ : the LOP is not satisfied.

\section{Example 2}

Trade balances are satisfied in any state of nature $s$ if $\sum_{i} c_{s}^{i *}=\sum_{i} e_{s}^{i}$.

Consider the same generic matrix of exchange rates (19) with $R_{s}^{1 \prime}=R_{s}^{1} / \epsilon_{s}^{1}$ and $R_{s}^{2 \prime}=R_{s}^{2} / \epsilon_{s}^{2}$ and $\tau_{s}^{2}=\epsilon_{s}^{1} / \epsilon_{s}^{2}$.

Using exactly the same arguments than in Example 1, we obtain $x_{1}^{1 *}=$ $x_{2}^{1 *}=x_{1}^{2 *}=x_{2}^{2 *}=0$. 
If this allocation is an equilibrium, it solves (3): there are positive multipliers $\mu^{i *}$ such that $q_{k}^{*}=\mu^{i *} \sum_{s} \pi_{s}^{i} u^{\prime}\left(c_{s}^{i *}\right) R_{s k}^{i \prime}$ for any $k$ and $i=1,2$. Noticing that $c_{s}^{i *}=e_{s}^{i}+R_{s 1}^{i \prime} x_{1}^{i *}+R_{s 2}^{i \prime} x_{2}^{i *}$ and $\left(c_{1}^{1}, c_{2}^{1}, c_{1}^{2}, c_{2}^{2}\right)^{*}=(1,1,1,2)$, we find the same $q_{1}^{*}$ 's as in Example 1 (see (20)), while the $q_{2}^{*}$ 's become now

$$
q_{2}^{*}=\frac{\mu^{1 *}}{2} \sum_{s} \frac{\pi_{s}^{1} R_{s 2}^{1}}{\sqrt{c_{s}^{1 *}}}=\frac{\mu^{1 *}}{2} \text { and } q_{2}^{*}=\frac{\mu^{2 *}}{2} \sum_{s} \frac{\pi_{s}^{2} R_{s 2}^{2 \prime}}{\sqrt{c_{s}^{2 *}}}=\frac{\mu^{2 *}}{2}\left(\frac{\tau_{1}^{2}}{2}+\frac{\tau_{2}^{2}}{\sqrt{2}}\right)
$$

But

$$
q_{1}^{*}=q_{2}^{*} \Leftrightarrow \frac{\mu^{2 *}}{2} \frac{\tau_{2}^{2}}{\sqrt{2}}=\frac{\mu^{2 *}}{2}\left(\frac{\tau_{1}^{2}}{2}+\frac{\tau_{2}^{2}}{\sqrt{2}}\right) \Leftrightarrow \tau_{1}^{2}=0
$$

which is impossible.

\section{References}

[1] Allouch, N., Le Van, C. and F.H. Page Jr. (2002). The geometry of arbitrage and the existence of competitive equilibrium, Journal of Mathematical Economics 38, 373-39.

[2] Dana R.A, and M. Jeanblanc (2003). Financial Markets in Continuous Time. Springer Finance Textbooks.

[3] Dana R.A, C. Le Van and F. Magnien (1999). On the different notions of arbitrage and existence of equilibrium. Journal of Economic Theory 86, 169-193.

[4] Frenkel J.A. and M.L. Mussa (1980). The efficiency of foreign exchange markets and measures of turbulence. American Economic Review 70, 374381.

[5] Hart O. (1974). On the existence of an equilibrium in a securities model. Journal of Economic Theory 9, 293-311.

[6] Page F.H. and M.H. Wooders (1996). A necessary and sufficient condition for compactness of individually rational and feasible outcomes and existence of an equilibrium. Economics Letters 52, 153-162.

[7] Rogoff K. (1996). The purchasing power parity puzzle. Journal of Economic Literature 34, 647-668.

[8] Werner J. (1987). Arbitrage and the existence of competitive equilibrium. Econometrica 55, 1403-1418. 